\title{
Combining Virtual Touch Tissue Imaging and BI-RADS May Improve Solid Breast Lesion Evaluation
}

\author{
Size Wu Xiaojing Cui Li Huang Xuefei Bai \\ Department of Ultrasound, The First Affiliated Hospital of Hainan Medical University, Haikou, China
}

\section{Keywords}

Breast lesions - Carcinoma - Ultrasound .

Ultrasound elastography - Acoustic radiation force impulse, ARFI - Virtual touch tissue imaging, VTI

\section{Summary}

Background: Ultrasound elastography (UE) is a novel imaging method. The purpose of this study was to determine the utility of Virtual Touch tissue imaging in the evaluation of solid breast lesions. Patients and Methods: 209 breast solid lesions in 192 patients that had been evaluated using ultrasound (US) and UE were reviewed and analyzed. Results: The sensitivity, specificity, accuracy, positive predictive value, and negative predictive value for UE, US, and US plus UE in the differentiation of malignant from benign breast lesions were 80.8, 75.6, $77.9,73.1$, and $82.8 \%$ for UE, 87.2, 86.1, 86.6, 83.7, and $89.2 \%$ for US, and $92.5,86.9,89.5,85.3$, and $93.4 \%$ for US plus UE. There were significant differences between UE and US plus UE (all $p<0.05$ ). Except for accuracy, there were no significant differences between UE and US or US and US plus UE (all $p>0.05$ ). The area under the curve obtained from the ROC curve for UE, US, and US plus UE in differentiating malignant from benign lesions was $0.845,0.884$, and 0.922 , respectively. Conclusion: The UE scoring system is not superior to BI-RADS in the sonographic evaluation of solid breast lesions. Combined use may improve the performance.

(c) 2017 S. Karger GmbH, Freiburg

\section{Introduction}

Ultrasonography (US) has emerged as the most important adjunct to mammography for the diagnosis of breast diseases with the Breast Imaging Reporting and Data System (BI-RADS). Ultrasound elastography (UE), which can examine tissue elasticity/ hardness, is used for the evaluation of breast diseases with a focus on the differentiation between benign and malignant breast lesions, based upon the clinical finding that malignant lesions of the breast are generally harder on palpation than normal breast tissue and benign lesions [1-9]. Published reports have shown that UE combined with US can reduce benign biopsies $[1,3]$. However, reports of the advantages and pitfalls of UE in the assessment of breast lesions have been discrepant [2, 6-8]. Therefore, summarization of experiences and further investigation of the role of UE in the assessment of breast lesions is necessary. The aim of this study was to determine the role and potential benefits of acoustic radiation force impulse (ARFI) Virtual Touch ${ }^{\mathrm{TM}}$ tissue imaging (VTI) (Siemens Medical Solutions, Mountain View, CA, USA) in the sonographic evaluation of solid breast lesions.

\section{Patients and Methods}

\section{Patients}

A database of patients with breast diseases, who were consecutively referred to our tertiary hospital and underwent simultaneous US and UE between May 2012 and March 2016, was retrospectively reviewed. Patients with solid lesions who underwent surgery or biopsy were included, and patients with pure cystic lesions or other diseases and those with solid lesions who did not undergo surgery or biopsy were excluded.

All procedures were in accordance with the ethical standards of the responsible committee on human experimentation (institutional and national) and with the World Medical Association Declaration of Helsinki (revised in 2000). An institutional review board approved this retrospective study, and neither patient approval nor informed consent were required for the review of US and UE images and common medical records.

\section{KARGER}

() 2017 S. Karger GmbH, Freiburg 


\section{US and UE Examinations}

US and UE examinations were performed by 6 radiologists with 5-21 years of experience in US examination of the breast and 6 months' experience in UE examination of the breast. All examinations were carried out using the ACUSON S2000 ${ }^{\mathrm{TM}}$ ultrasound system (Siemens Medical Solutions) equipped with an L5 linear transducer (6-14 MHz). US evaluation of the breast was carried out at a frequency of 11-14 MHz at first, focusing on focal lesions. The sonographic characteristics of the focal lesions, such as size, shape, echogenicity, margin, and posterior acoustic effect, were scrutinized. UE was subsequently carried out. UE images were obtained by applying the linear transducer very lightly to the skin over the targeted lesion using an adequate amount of coupling gel. The probe was held still for a few seconds to generate several UE images. The procedure was repeated 3 times, and an image presenting adequate quality (quality factor $>50$ ) was saved. US and UE images were simultaneously displayed in a split screen mode, with the US images on the left and the semitransparent color-scale UE images superimposed on the corresponding US images on the right. An appropriate rectangular region of interest was set for UE acquisition. Obtained images that showed color homogeneity within the region of interest were displayed on the screen. Each pixel of the elasticity image was shown as 1 of 256 specific colors, representing the extent of strain. The scale ranged from red, which showed areas of greatest strain (i.e., softest component) to blue, which showed no strain (i.e., hardest component).

Representative images of solid lesions obtained by US and UE were selected and saved in the picture archiving and communication system (PACS).

\section{Image Analysis}

US and UE images of solid breast lesions were sorted, filed, and numbered by 2 radiologists who did not participate in the US and UE examinations of the patients. 209 solid breast lesions (94 malignant, 115 benign) in 192 patients were selected. The mean size of the breast lesions was $22.99 \pm 5.94 \mathrm{~mm}$ (malignant lesions $18.16 \pm 5.19 \mathrm{~mm}$, benign lesions $24.02 \pm 5.40 \mathrm{~mm}$ ), with a range of 6-39 mm. The mean age of the 192 patients was 46 years (range $20-85$ years). The images were studied by 2 radiologists with 3 years' experience in breast UE and 13 and 17 years' experience in breast US, respectively. Both readers were blinded to patient identification, clinical history, other imaging results, pathological findings, and the final diagnosis. First, the 2 readers analyzed the images independently for US and UE, then they discussed each case with each other and reached the final results with consensus. The breast lesions were assessed according to BI-RADS based on US first, and the 5-point scoring system for UE second. The UE characteristics were analyzed with reference to the 5-point scoring system proposed by Zhi et al. [2]: A score of 1 indicated even strain for the entire lesion (i.e., the entire lesion was evenly shaded in green); a score of 2 indicated strain in the majority of the lesion with focal no-strain spots (i.e., the lesion was shaded in green with focal blue spots); a score of 3 indicated strain and no strain in half of the lesion each (i.e., the lesion was half green and half blue); a score of 4 indicated no or little strain in the entire lesion (i.e., the entire lesion was blue or predominantly blue with a little green); and a score of 5 indicated no or little strain in the entire lesion and the surrounding area (i.e., the entire lesion and its surrounding area were blue or blue with focal green spots). An analysis on a per lesion basis was performed. A BI-RADS category was applied according to the representative US characteristics of the lesion (benign lesions BI-RADS 2 and 3, malignant lesions BI-RADS 4 and 5). The highest reproducible UE score was adopted as the elastography score of the lesion (see supplementary fig. 1 for an example of confirmed breast carcinoma, www.karger.com/?DOI=456026). Based on the 5-point scoring system for UE, breast lesions scored 1,2, and 3 were assumed to be benign; those scored 4 and 5 were assumed to be malignant. The final BI-RADS category was modified after combining the UE scoring system and BI-RADS. If a nodule was substantially less stiff on UE, the initial BIRADS category was downgraded after referring to US findings; if the nodule presented substantially stiffer on UE than expected from the corresponding initial BI-RADS category, the category was upgraded after referring to US findings. The presumed diagnosis was compared with the final diagnosis made on the basis of histopathologic findings.
Table 1. Histopathologic diagnoses of malignant and benign breast lesions

\begin{tabular}{lr}
\hline & $\mathrm{n}$ \\
\hline Malignant lesions ( $\mathrm{n}=94)$ & 8729 \\
Infiltrative ductal carcinoma & 2 \\
Ductal carcinoma in situ & 2 \\
Squamous cell carcinoma & 3 \\
Mucinous carcinoma & \\
Benign lesions (n = 115) & 36 \\
Hyperplasia & 54 \\
Fibroadenoma & 6 \\
Intraductal papilloma & 2 \\
Sclerosing adenosis & 5 \\
Chronic inflammation & 12 \\
Other solid benign lesions & \\
\hline
\end{tabular}

\section{Statistical Analysis}

The presumed diagnosis was compared with the final diagnosis with respect to sensitivity, specificity, and area under the curve (AUC) using a receiver operating characteristic (ROC) curve analysis for the diagnostic performance of US, UE, and US plus UE in differentiating benign from malignant lesions. Sensitivity, specificity, accuracy, positive predictive value (PPV), and negative predictive value (NPV) of US, UE, and US plus UE were calculated, and Pearson's $\chi^{2}$ test was used for analysis of comparison. A p value (two-sided) of less than 0.05 was considered to indicate a statistically significant difference. In this study, the US and UE images were evaluated by 2 readers in consensus; therefore, the interobserver agreement for BI-RADS category and UE score was not estimated. The statistical analyses were performed by using SPSS 20 (SPSS Corp., IBM, Armonk, NY, USA)

\section{Results}

The histopathologies and numbers of benign and malignant breast lesions are listed in table 1. The sensitivity, specificity, accuracy, PPV, and NPV for US, UE, and US plus UE in the differentiation of malignant from benign breast lesions are listed in table 2. The AUC obtained from the ROC curve for the UE scores, US BI-RADS, and US plus UE in differentiating malignant from benign lesions was $0.845,0.884$, and 0.922 , respectively. Supplementary figures 1-4 (www.karger.com/?DOI=456026) illustrate the UE and US characteristics of both benign and malignant breast lesions.

\section{Discussion}

The histopathologic nature of a breast lesion may be revealed by measuring its elasticity [1-4]. US imaging systems specially equipped with elastography features such as VTI allow the investigation of elastic tissue properties on the basis of UE. Our results for specificity, sensitivity, and accuracy of UE were 75.6, 80.8, and $77.9 \%$, respectively, much lower (except for sensitivity) than those reported by Zhi et al. [2] (86.4, 80.8, and 83.5\%) and Xue et al. [5] $(85.1,93.6$, and $89.7 \%)$. Both the specificity and accuracy of UE were lower than those of US in our study, which is in contrast to the findings of Zhi et al. [2] and Xue et al. [5] according to which the specificity and accuracy of UE were higher than those of US. The AUC of UE was 0.845 in our study, which was lower than that found by Zhi et al. [2] (0.86) and Xue et al [5] (0.93). The specificity, sensitivity, 
Table 2. Sensitivity, specificity, accuracy, positive predictive value (PPV), and negative predictive value (NPV) for ultrasound elastography (UE), ultrasound (US), and US plus UE in differentiating malignant from benign breast lesions

\begin{tabular}{llllll}
\hline Variables & Sensitivity, \% & Specificity, \% & Accuracy, $\%$ & PPV, \% & NPV, \% \\
\hline UE & $80.8(76 / 94)$ & $75.6(87 / 115)$ & $77.9(163 / 209)$ & $73.1(76 / 104)$ & $82.8(87 / 105)$ \\
US & $87.2(82 / 94)$ & $86.1(99 / 115)$ & $86.6(181 / 209)$ & $83.7(82 / 98)$ & $89.2(99 / 111)$ \\
US plus UE & $92.5(87 / 94)$ & $86.9(100 / 115)$ & $89.5(187 / 209)$ & $85.3(87 / 102)$ & $93.4(100 / 107)$ \\
p value & $0.319^{\mathrm{a}}$ & $0.064^{\mathrm{a}}$ & $0.029^{\mathrm{a}}$ & $0.088^{\mathrm{a}}$ & $0.238^{\mathrm{a}}$ \\
& $0.030^{\mathrm{b}}$ & $0.042^{\mathrm{b}}$ & $0.002^{\mathrm{b}}$ & $0.039^{\mathrm{b}}$ & $0.019^{\mathrm{b}}$ \\
& $0.333^{\mathrm{c}}$ & $1.000^{\mathrm{c}}$ & $0.451^{\mathrm{c}}$ & $0.846^{\mathrm{c}}$ & $0.339^{\mathrm{c}}$ \\
\hline
\end{tabular}

${ }^{a}$ Indicates UE versus US.

bIndicates UE versus US plus UE.

'Indicates US versus US plus UE. accuracy, and PPV of US plus UE were higher than those of UE and US in our study. These results indicate that the combining use of US and UE can improve the diagnostic performance, while UE alone shows no trend to improving the diagnostic performance.

In our opinion, the lower specificity, sensitivity, accuracy, and PPV of UE compared to US in this study are mainly due to the high variability of the UE examinations, and may also involve several other factors such as component of the breast lesion, histopathologic pattern, location, depth and size of the breast lesion, breast thickness, patient age, and different ultrasound systems and image interpretation (Zhou et al [10]). Women's breasts in our ethnic and geographic region are small [2], so thickness and depth did not have a negative affect on the accuracy of UE. We noted a clustering effect in this study in that some patients presented with benign and malignant lesion synchronously, and some patients had several different benign lesions, e.g., 2 patients with fibroadenoma and infiltrative ductal carcinoma in the same breast and 3 patients with fibroadenoma and hyperplasia in the same breast, which adds difficulty to the US and UE evaluation.

Generally, benign lesions of the breast are harder than normal breast tissue but softer than malignant tumors [4-6, 11]; however, there are exceptions. False-positive readings may be influenced by benign conditions that display poor elasticity such as scar tissue, hyperplasia, and fibroadenomas with a predominant fibrous component. Hyalinized fibroadenomas, radial scars, fat necrosis, hyperplasia, and complicated cysts frequently present with a bizarre shade on UE $[8,12,13]$. Invasive ductal carcinoma and fibroadenoma are different pathologic entities; however, their UE appearance may be similar with a score of 5 , while on US their characteristics may clearly support their pathologies. As a results, more breast lesions assessed with BI-RADS are consistent with the final diagnosis than those scored with the UE system. Similarly, some malignant lesions appear like benign lesion on US, causing falsenegative BI-RADS results.

Sometimes breast hyperplasia is difficult to distinguish from malignant lesions on US, and it may be scored as 2, 3, 4, or 5 on UE in different cases. In this series, 9 cases of hyperplasia were assigned to BI-RADS category $4 \mathrm{a}$, while on UE they were scored as 4 . As a result, they received a presumed diagnosis of malignancy and increased the false-positive rate.

Mucinous carcinoma would be assumed to be soft because it is rich in mucin; however, it is in fact found to be harder and scored 5 in this series as shown in supplementary figure 3 (www.karger. com/?DOI=456026). This is consistent with reports by Grajo and Barr [14] and Mori et al. [15] that mucinous carcinoma presents relatively harder on UE.

Some ductal papillomas had some fluid around the tumor; however, it was not shaded authentically on UE as shown on supplementary figure 4 (www.karger.com/?DOI=456026). The anechoic liquid around the tumor was shaded blue, which was not consistent with the sonographic finding and pathology. This suggests that small heterogeneous areas within an entity may not be well shaded on UE or present as an artifact, which may influence the interpretation of the lesion and increase the false-positive rate. Au et al. [3] reported that UE could help downgrade BI-RADS category 4 and reduced biopsy. In this study, 12 cases of BI-RADS category 3 were upgraded to category 4 , and 6 cases of BI-RADS category 4 were downgraded to category 3; a new diagnosis based on US plus UE was established, and the sensitivity and specificity improved.

Based on these results, we believe that the findings of UE can only complement the BI-RADS of US if UE reveals the authentic elasticity of a lesion.

Potential biases in our study are that only few BI-RADS 2 patients underwent surgery or biopsy, patients who did not have histopathology were not included, lesions with a size of $<6 \mathrm{~mm}$ in the database were not included, and pure cystic lesions were not included.

\section{Conclusion}

In conclusion, our clinical experience is that VTI and the UE scoring system are not superior to BI-RADS in the sonographic evaluation of solid breast lesions in terms of sensitivity, specificity, and accuracy; however, the UE scoring system can provide some additional information, and US plus UE can improve the performance of breast evaluations.

\section{Online Supplementary Figures}

Supplementary Fig. 1. Image of a fibroadenoma. BI-RADS category 3 and UE score 3.

Supplementary Fig. 2. Image of an invasive ductal carcinoma. BI-RADS category 5 and UE score 5. 
Supplementary Fig. 3. Image of a mucinous carcinoma. BI-RADS category 3 and UE score 5.

Supplementary Fig. 4. Image of a ductal papilloma. BI-RADS category 3 and UE score 4.

To access the supplementary figures, please refer to www.karger.com/? $D O I=456026$.

\section{Disclosure Statement}

All authors declare that there is no conflict of interest.

\section{References}

1 Ianculescu V, Ciolovan LM, Dunant A, Vielh P, Mazouni C, Delaloge S, Dromain C, Blidaru A, Balleyguier C: Added value of Virtual Touch IQ shear wave elastography in the ultrasound assessment of breast lesions. Eur J Radiol 2014;83:773-777.

2 Zhi H, Ou B, Xiao X, Peng YL, Wang Y, Liu LS, Xiao Y, Liu SJ, Wu CJ, Jiang YX, Parajuly SS, Xu P, Hao Y, Li J, Luo BM: Ultrasound elastography of breast lesions in Chinese women: a multicenter study in China. Clin Breast Cancer 2013;13:392-400.

3 Au FW, Ghai S, Moshonov H, Kahn H, Brennan C, Dua H, Crystal P: Diagnostic performance of quantitative shear wave elastography in the evaluation of solid breast masses: determination of the most discriminatory parameter. Am J Roentgenol 2014;203:W328-336.

4 Doherty J, Trahery G, Nightingale K, Palmeri ML Acoustic radiation force elasticity imaging in diagnostic ultrasound. IEEE Trans Ultrason Ferroelectr Freq Control 2013;60:685-701.

5 Xue Zhang, Yang Xiao, Jie Zeng, Qiu W, Qian M, Wang C, Zheng R, Zheng H: Computer-assisted assessment of ultrasound real-time elastography: initial experience in 145 breast lesions. Eur J Radiol 2014. 83:e1-e7.
6 Kim YS, Park JG, Kim BS, Lee CH, Ryu DW: Diagnostic value of elastography using acoustic radiation force impulse imaging and strain ratio for breast tumors. J Breast Cancer 2014;17:76-82.

7 Meng W, Zhang G, Wu C, Wu G, Song Y, Lu Z: Preliminary results of acoustic radiation force impulse (ARFI) ultrasound imaging of breast lesions. Ultrasound Med Biol 2011;37:1436-1443.

8 Barr RG, Destounis S, Lackey LB 2nd, Svensson WE, Balleyguier C, Smith C: Evaluation of breast lesions using sonographic elasticity imaging: a multicenter trial. J Ultrasound Med 2012;31:281-287.

9 Destounis S, Arieno A, Morgan R, Murphy P, Seifert P, Somerville $\mathrm{P}$, Young W: Clinical experience with elasticity imaging in a community-based breast center. J Ultrasound Med 2013;32:297-302.

10 Zhou J, Zhan W, Chang C, Zhang X, Jia Y, Dong Y, Zhou C, Sun J, Grant EG: Breast lesions: evaluation with shear wave elastography, with special emphasis on the 'stiff rim' sign. Radiology 2014;272:63-72.
11 Li G, Li DW, Fang YX, Song YJ, Deng ZJ, Gao J, Xie Y, Yin TS, Ying L, Tang KF: Performance of shear wave elastography for differentiation of benign and malignant solid breast masses. PLoS One 2013;8:e76322.

12 Thomas A, Degenhardt F, Farrokh A, Wojcinski S, Slowinski T, Fischer T: Significant differentiation of focal breast lesions: calculation of strain ratio in breast sonoelastography. Acad Radiol 2010;17:558-563.

13 Athanasiou A, Tardivon A, Tanter M, Sigal-Zafrani B, Bercoff J, Deffieux T, Gennisson JL, Fink M, Neuenschwander S: Breast lesions: quantitative elastography with supersonic shear imaging - preliminary results. Radiology 2010;256:297-303.

14 Grajo JR, Barr RG: Strain elastography for prediction of breast cancer tumor grades. J Ultrasound Med 2014; 33:129-134.

15 Mori M, Tsunoda H, Kawauchi N, Kikuchi M, Honda S, Suzuki K, Yamauchi H: Elastographic evaluation of mucinous carcinoma of the breast. Breast Cancer 2012; 19:60-63. 